\title{
Perfil nutricional e estilo de vida de pacientes pré e pós-cirurgia bariátrica
}

\author{
Nutritional profile and lifestyle of patients before and after bariatric surgery
}

\author{
Letícia Tomicki Zyger ${ }^{1} \bowtie$, Vivian Polachini Skzypek Zanardo', Camila Tomicki² \\ ${ }^{1}$ Universidade Regional Integrada do Alto Uruguai e das Missões (URI). Erechim, RS. \\ 2 Programa de Pós-Graduação em Educação Física da Universidade Federal de Santa Catarina (UFSC). Florianópolis, SC.
}

\section{RESUMO}

Objetivos: Analisar o perfil nutricional e o estilo de vida de pacientes antes e após a cirurgia bariátrica por técnica de derivação gástrica em Y de Roux.

Métodos: Estudo de coorte retrospectivo realizado no período de 2010 a 2014, incluindo dados de pacientes submetidos à cirurgia bariátrica por derivação gástrica em Y de Roux em um hospital privado, localizado em um município da região norte do Rio Grande do Sul. A análise considerou dados sociodemográficos, estilo de vida, antropometria e consumo alimentar de calorias, nutrientes, vitaminas e sais minerais antes e um ano após a cirurgia. Foram analisados também os resultados de exames bioquímicos (vitamina B12, hemoglobina, albumina, hematócrito e ferritina) no período de seis meses a um ano após a cirurgia.

Resultados: Foram incluídos 50 pacientes, sendo 44 (88\%) do sexo feminino. Trinta e cinco (70\%) pacientes apresentavam patologias antes da cirurgia, sendo as mais frequentes hipertensão arterial (35\%) e diabetes mellitus (20\%). Na comparação entre a avaliação pré-cirúrgica e a

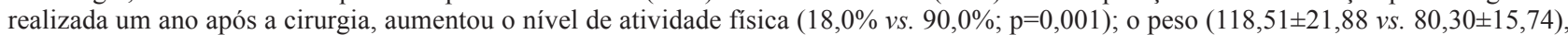

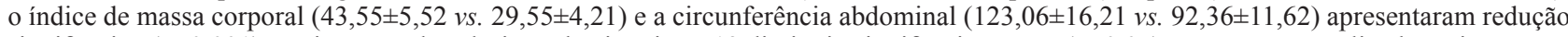
significativa $(\mathrm{p}<0,001)$; e a ingestão de calorias e de vitamina B12 diminuiu significativamente $(\mathrm{p}<0,05)$. Nos exames realizados seis meses a um ano após a cirurgia, um paciente apresentou deficiência de Vitamina B12, sete apresentaram anemia e três deficiência de ferritina.

Conclusões: Após a derivação gástrica em Y de Roux houve perda de peso, redução do índice de massa corporal e da circunferência abdominal e diminuição da taxa de sedentarismo. Foram detectadas algumas deficiências nutricionais, o que indica que há necessidade de acompanhamento do estado nutricional no pós operatório de cirurgia bariátrica, para garantir o sucesso do tratamento.

DESCRITORES: obesidade; cirurgia bariátrica; estado nutricional; deficiências nutricionais.

\section{ABSTRACT}

Aims: To analyze the nutritional profile and the lifestyle of patients before and after bariatric surgery by Roux-en-Y gastric bypass.

Methods: Retrospective cohort study carried out in the period from 2010 to 2014, including data on patients submitted to bariatric surgery by Roux-en-Y gastric bypass in a private hospital located in the northern region of Rio Grande do Sul, Brazil. Sociodemographic data, lifestyle, anthropometric measurements, and the intake of calories, nutrients, vitamins, and mineral salts before surgery and one year thereafter were analyzed. The results for biochemical tests (vitamin B12, hemoglobin, albumin, hematocrit, and ferritin) performed six months to one year after surgery were also assessed.

Results: Fifty patients were included in the study, and $44(88 \%)$ were female. Thirty-five patients (70\%) presented with some conditions before surgery, among which high blood pressure (35\%) and diabetes mellitus $(20 \%)$ were the most frequent. Between the preoperative evaluation and one year after the surgery, physical activity level increased $(18.0 \% v s .90 .0 \%$; $\mathrm{p}=0.001)$ whereas weight $(118.51 \pm 21.88 v s .80 .30 \pm 15.74)$, body mass index (43.55 $\pm 5.52 v s .29 .55 \pm 4.21)$, and abdominal circumference (123.06 $\pm 16.21 v s .92 .36 \pm 11.62)$ decreased significantly ( $<<0.001)$. The intake of calories and of vitamin B12 also decreased significantly $(\mathrm{p}<0.05)$. The tests performed within six months to one year after the surgery indicated one patient with vitamin B12 deficiency, seven patients with anemia, and three with ferritin deficiency.

Conclusions: After bariatric surgery by Roux-en-Y gastric bypass there was weight loss, reduction in body mass index and abdominal circumference, and a decrease in sedentary lifestyle. Some nutritional deficiencies were observed, which indicates the need for nutritional management after bariatric surgery in order to ensure a successful outcome.

KEY WORDS: obesity; bariatric surgery; nutritional status; deficiency diseases. 
Abreviaturas: CA, circunferência abdominal; IDR, ingestão diária recomendada; DGYR, derivação gástrica em Y de Roux; DP, desvio padrão; IMC, índice de massa corporal; Máx., máximo; Mín., mínimo; ng, nanograma; pg, picograma; URI, Universidade Regional Integrada do Alto Uruguai e das Missões; OMS, Organização Mundial da Saúde.

\section{INTRODUÇÃO}

A obesidade é uma doença crônica causada por múltiplos fatores, sendo o excesso de gordura corporal sua principal característica. No mundo existem cerca de 250 milhões de pessoas sofrendo com sobrepeso ou obesidade, e o Brasil está incluso nesta estatística, com cerca de 82 milhões de pessoas apresentando essa condição [1-3].

O levantamento mais recente realizado pela Vigilância de Fatores de Risco e Proteção para Doenças Crônicas por Inquérito Telefônico (Vigitel) aponta que houve um aumento do sobrepeso e da obesidade e, com isso, aumentou o risco de doenças crônicas entre os brasileiros. De todos os entrevistados no País, 20\% relataram ter o colesterol alto, sendo que esse relato foi mais frequente entre as mulheres $(22,2 \%)$ do que entre os homens (17,6\%). A obesidade se torna mais comum com o avanço da idade e em pessoas que apresentam menor nível de escolaridade [2]. De acordo com o Instituto Brasileiro de Geografia e Estatística [4], $63 \%$ da população do Rio Grande do Sul está acima do peso. Como fatores contribuintes nesse estado do sul do Brasil, poderiam ser citados a cultura e o tipo de gastronomia, como também o clima frio em boa parte do ano, que contribui para o sedentarismo [4].

O tratamento da obesidade deve ser conduzido da mesma forma como nas outras doenças crônicas, através de reeducação alimentar e atividade física. A obesidade pode ser uma doença silenciosa, mas geralmente está associada a outras doenças. Os melhores resultados para o seu tratamento são alcançados lentamente; a busca por tratamentos milagrosos e resultados rápidos aumentam a chance de abandono do tratamento e a tendência a recidivas [5]. É consenso que planos alimentares com redução moderada de calorias, dentro de metas reais e sustentáveis, associados à prática de atividade física regular e orientada, são a melhor opção de controle nutricional da obesidade [5].

O índice de massa corporal (IMC) igual ou acima de $40 \mathrm{~kg} / \mathrm{m}^{2}$ (obesidade mórbida) indica diminuição da expectativa de vida [6] e aumento da mortalidade por doença cardiovascular. Nos casos graves de obesidade, onde houve falha na adesão ao tratamento clínico, a cirurgia bariátrica é um método eficiente, reduzindo a mortalidade e promovendo melhora clínica das comorbidades [7]. Segundo a Sociedade Brasileira de Cirurgia Bariátrica e Metabólica, em 2003 no Brasil foram realizadas 16 mil cirurgias bariátricas, enquanto em 2010 foram realizadas 60 mil cirurgias, 35\% delas por videolaparoscopia [8].

A escolha pela cirurgia bariátrica para tratamento da obesidade deve ser avaliada com muita cautela, pois os indivíduos após o procedimento cirúrgico podem apresentar deficiências nutricionais, incluindo deficiências de ferro, cálcio, vitamina B12, vitamina $\mathrm{D}$, acido fólico, zinco e albumina. É muito importante que o paciente apresente compromisso com resultados e que mantenha acompanhamento com uma equipe multiprofissional. Com orientações sobre consumo de suplementos, pode-se prevenir problemas nutricionais e metabólicos [9-11].

A técnica de gastroplastia com derivação gastrojejunal, conhecida por derivação gástrica em Y de Roux (DGYR) é a mais utilizada atualmente. Os resultados da DGYR mostram eficácia na perda de peso, assim como na redução das comorbidades e melhora da qualidade de vida. Essa técnica exibe alguns dos resultados mais consistentes em longo prazo [12-14].

Diante deste contexto, este estudo teve como objetivo analisar o perfil nutricional e alguns dados do estilo de vida de pacientes antes e após cirurgia bariátrica.

\section{MÉTODOS}

Foi realizado um estudo de coorte retrospectivo, com pacientes submetidos à cirurgia bariátrica em um hospital privado, localizado em um município da região Norte do Rio Grande do Sul, Brasil. Foram coletados dados dos prontuários da equipe multidisciplinar de cirurgia bariátrica, que presta atendimento pré e pós-operatório aos pacientes que realizam esse procedimento na instituição, no período de 2010 a 2014, sendo incluídos todos os indivíduos de ambos os sexos, com idade entre 18 e 66 anos, que realizaram o procedimento DGYR com um mesmo cirurgião.

Os seguintes dados foram levantados antes e após um ano da cirurgia bariátrica: sociodemográficos sexo, idade, escolaridade e renda; relacionados ao estilo de vida - atividade física, ingestão de bebida alcoólica, tabagismo e hábitos alimentares; antropométricos - estatura, peso, IMC e circunferência abdominal (CA); relacionados ao consumo alimentar de macro e micronutrientes, sendo considerada a ingestão de alimentos e suplementos nutricionais. Foram analisados também os resultados dos últimos exames 
bioquímicos, coletados dos prontuários, realizados no período de 6 a 12 meses após a cirurgia: vitamina B12, ferritina, albumina, hematócrito e hemoglobina.

Para a classificação do IMC foram utilizados os parâmetros conforme a Organização Mundial da Saúde (OMS) [15]: IMC entre 18,5 kg/m² e 24,9 kg/m², eutrofia; IMC $\geq 25 \mathrm{~kg} / \mathrm{m}^{2}$, sobrepeso; e IMC $\geq 30 \mathrm{~kg} / \mathrm{m}^{2}$, obesidade. Os valores de circunferência abdominal foram considerados como risco de complicações metabólicas associadas à obesidade segundo a OMS [16]: aumentado (homem $\geq 94 \mathrm{~cm}$, mulher $\geq 80 \mathrm{~cm}$ ); muito aumentado (homem $\geq 102 \mathrm{~cm}$, mulher $\geq 88 \mathrm{~cm}$ ). Os valores recomendados de micronutrientes foram avaliados segundo a Ingestão Diária Recomendada (IDR) [17,18], sendo utilizado o software Avanutri ${ }^{\circledR}$ para o cálculo dos recordatórios de 24 horas antes e após seis meses da realização da cirurgia bariátrica. Para os exames bioquímicos, consideraram-se como referência os valores para vitamina B12, ferritina, albumina, hematócrito e hemoglobina conforme Resende et al. [19].

Para a estruturação do banco de dados utilizou-se o aplicativo Excel 2010 e para as análises o software Bio Estat, versão 5.0. As variáveis quantitativas foram expressas como média \pm desvio padrão. As variáveis qualitativas foram expressas como frequência absoluta e relativa. A distribuição da normalidade dos dados foi verificada por meio do teste de ShapiroWilk. As possíveis diferenças das variáveis IMC não categorizado, CA não categorizada, exames bioquímicos, macro e micronutrientes antes e após a cirurgia bariátrica foram verificadas utilizando o teste $\mathrm{t}$ de Student pareado para variáveis contínuas ou teste Mc Nemar para variáveis dicotômicas. Para investigar a relação existente entre percentual de perda de peso (calculado a partir da porcentagem de peso e CA original) e deficiências nutricionais; IMC e deficiências nutricionais; CA e deficiências nutricionais, utilizou-se o teste qui-quadrado de Pearson. Consideraram-se como estatisticamente significativos os resultados com $p<0,05$.

A pesquisa foi aprovada pelo Comitê de Ética em Pesquisa com Seres Humanos da Universidade Regional Integrada do Alto Uruguai e das Missões (URI) Erechim, sob protocolo no 868.709.

\section{RESULTADOS}

Foram coletados dados de 50 indivíduos, sendo $44(88 \%)$ do sexo feminino. A idade média dos participantes foi de $38,1 \pm 11,9$ anos, sendo o mínimo 18 anos e o máximo 66 anos. Em relação à renda, $25(50 \%)$ dos pacientes recebiam quatro salários mínimos, e quanto à escolaridade prevaleceu o ensino médio completo (40\%), seguido do ensino superior completo (34\%). Trinta e cinco (70\%) entre os pacientes avaliados apresentavam uma ou mais patologias antes da cirurgia bariátrica, prevalecendo hipertensão arterial sistêmica (35\%) e diabetes mellitus (20\%), sendo que $15 \%$ apresentavam as duas morbidades.

A Tabela 1 apresenta a descrição das variáveis dicotômicas antes e após a cirurgia bariátrica: atividade física, consumo de álcool e tabagismo. Houve diminuição significativa do número de pacientes sedentários $(\mathrm{p}=0,001)$.

Tabela 1. Descrição dos dados de estilo de vida em 50 pacientes que realizaram cirurgia bariátrica por derivação gástrica em Y de Roux, no período de 2010 a 2014. Rio Grande do Sul, Brasil.

\begin{tabular}{lccc}
\hline \multicolumn{1}{c}{ Variáveis } & $\begin{array}{c}\text { Sim } \\
\mathbf{n}(\%)\end{array}$ & $\begin{array}{c}\text { Não } \\
\mathbf{n}(\%)\end{array}$ & $\mathbf{p}^{*}$ \\
\hline $\begin{array}{l}\text { Sedentarismo } \\
\text { Pré }\end{array}$ & $41(82,0)$ & $09(18,0)$ & \\
$\quad$ Pós & $05(10,0)$ & $45(90,0)$ & $\mathbf{0 , 0 0 1}$ \\
Consumo de álcool & & & \\
Pré & $01(2,0)$ & $49(98,0)$ & 1,000 \\
$\quad$ Pós & $00(0,0)$ & $50(100,0)$ & \\
Tabagismo & & & \\
Pré & $00(0,0)$ & $50(100,0)$ & \\
Pós & $00(0,0)$ & $50(100,0)$ & \\
\hline
\end{tabular}

* Teste de McNemar.

Pré: antes da cirurgia; Pós: um ano após a cirurgia.

A Tabela 2 apresenta os valores médios dos exames bioquímicos realizados depois da cirurgia bariátrica. Apenas um paciente apresentou deficiência de vitamina B12; ao ser avaliado, nesse paciente, o aporte alimentar de vitamina B12 após a cirurgia, verificou-se que a oferta estava insuficiente.

Conforme os dados antropométricos avaliados antes e após um ano da cirurgia, observou-se que as médias de peso, IMC e CA apresentaram redução significativa $(\mathrm{p}=0,001)$ (Tabela 3). Na Tabela 4 estão descritas as variáveis categorizadas IMC e CA antes e depois da cirurgia. Em relação ao IMC, observou-se que em 31 pacientes $(62 \%)$ o diagnóstico após a cirurgia foi mudado para sobrepeso $(27,54 \%)$ e eutrofia $(4,8 \%)$. Com relação a CA, sete $(14 \%)$ dos pacientes apresentaram uma redução para níveis normais. Porém, 43 (86\%) ainda apresentam algum risco de complicações associadas à obesidade. A análise de correlação de Pearson revelou que houve uma correlação estatisticamente significativa entre perda de peso e diminuição da CA; $r(48)=0,530$ $(p<0,005)$, calculados em porcentagem a partir dos valores pré-cirúrgicos. 
Tabela 2. Resultados dos exames bioquímicos realizados entre seis meses e um ano após cirurgia bariátrica por derivação gástrica em Y de Roux, no período de 2010 a 2014 (n=50). Rio Grande do Sul, Brasil.

\begin{tabular}{|c|c|c|c|c|c|c|}
\hline \multirow{2}{*}{ Variáveis } & \multirow{2}{*}{ Mínimo } & \multirow{2}{*}{ Máximo } & \multirow{2}{*}{ Média $\pm D P$} & \multicolumn{3}{|c|}{$\begin{array}{l}\text { Classificação dos resultados dos } 50 \text { pacientes } \\
\text { em relação aos valores de referência* }\end{array}$} \\
\hline & & & & $\begin{array}{l}\text { Deficiente } \\
\text { n (\%) }\end{array}$ & $\begin{array}{l}\text { Normal } \\
\text { n (\%) }\end{array}$ & $\begin{array}{l}\text { Superior } \\
\text { n (\%) }\end{array}$ \\
\hline Vitamina B12 (pg/ml) & 103,0 & 1862,0 & $340,8 \pm 256,7$ & $01(2)$ & $44(88)$ & $05(20)$ \\
\hline Hemoglobina (g/dl) & 11 & 17,3 & $13,2 \pm 1,2$ & $07(14)$ & $42(84)$ & $01(2)$ \\
\hline Albumina (g/dl) & 3,1 & 41,0 & $4,6 \pm 5,2$ & $00(0)$ & $48(96)$ & $02(4)$ \\
\hline Hematócrito (\%) & 34 & 126 & $41,9 \pm 12,8$ & $03(6)$ & $45(90)$ & $02(4)$ \\
\hline Ferritina $(\mathrm{ng} / \mathrm{ml})$ & 7,3 & 2756,0 & $201,7 \pm 389,9$ & $03(6)$ & $31(62)$ & $16(32)$ \\
\hline
\end{tabular}

DP, desvio padrão; $\mathrm{pg} / \mathrm{ml}$, picograma por mililitro; $\mathrm{g} / \mathrm{dl}$, grama por decilitro; $\mathrm{ng} / \mathrm{ml}$, nanograma por mililitro.

* Valores de referência conforme Resende et al. [19].

Tabela 3. Dados antropométricos obtidos antes e um ano após cirurgia bariátrica por derivação gástrica em Y de Roux, no período de 2010 a 2014 ( $n=50)$. Rio Grande do Sul, Brasil.

\begin{tabular}{lcccc}
\hline \multicolumn{1}{c}{ Variáveis } & Mínimo & Máximo & Média \pm DP & $\mathbf{p}^{*}$ \\
$\begin{array}{l}\text { Peso }(\mathrm{kg}) \\
\text { Pré }\end{array}$ & 90 & 184 & $118,51 \pm 21,88$ & \\
$\quad$ Pós & 55 & 144 & $80,30 \pm 15,74$ & $\mathbf{0 , 0 0 1}$ \\
$\begin{array}{l}\text { IMC }\left(\mathrm{kg} / \mathrm{m}^{2}\right) \\
\text { Pré }\end{array}$ & 34,5 & 56,4 & $43,55 \pm 5,52$ & $\mathbf{0 , 0 0 1}$ \\
Pós & 22,1 & 40,8 & $29,55 \pm 4,21$ & \\
CA (cm) & & & & \\
Pré & 90 & 165 & $123,06 \pm 16,21$ & $\mathbf{0 , 0 0 1}$ \\
Pós & 68 & 124 & $92,36 \pm 11,62$ & \\
\hline
\end{tabular}

* Teste t de Student.

DP, desvio padrão; IMC, índice de massa corporal; CA, circunferência abdominal.

Pré: antes da cirurgia; Pós: um ano após a cirurgia.

Tabela 4. Índice de massa corporal e circunferência abdominal antes e um ano após cirurgia bariátrica por derivação gástrica em Y de Roux, no período de 2010 a $2014(\mathrm{n}=50)$. Rio Grande do Sul, Brasil.

\begin{tabular}{lcc}
\hline \multicolumn{1}{c}{ Variáveis } & $\begin{array}{c}\text { Pré cirurgia } \\
\mathbf{n}(\%)\end{array}$ & $\begin{array}{c}\text { Pós cirurgia } \\
\mathbf{n}(\%)\end{array}$ \\
\hline $\begin{array}{l}\text { IMC categorizado }\left(\mathrm{kg} / \mathrm{m}^{2}\right)^{*} \\
\text { Baixo peso }\end{array}$ & 0 & 0 \\
Peso normal & 0 & $04(8)$ \\
Sobrepeso & 0 & $27(54)$ \\
Obesidade & $50(100)$ & $19(38)$ \\
CA categorizado $(\mathrm{cm})^{*}$ & & \\
$\quad$ Normal & 0 & $07(14)$ \\
Risco elevado & 0 & $17(34)$ \\
Risco muito elevado & $50(100)$ & $26(52)$ \\
\hline
\end{tabular}

IMC, índice de massa corporal; CA, circunferência abdominal.

Peso normal: $18,5 \mathrm{~kg} / \mathrm{m}^{2}$ a $24,9 \mathrm{~kg} / \mathrm{m}^{2}$; sobrepeso: $\geq 25 \mathrm{~kg} / \mathrm{m}^{2}$; obesidade: $\geq 30 \mathrm{~kg} / \mathrm{m}^{2}$

Circunferência abdominal - risco elevado: homem $\geq 94 \mathrm{~cm}$, mulher $\geq 80 \mathrm{~cm}$; risco muito elevado: homem $\geq 102 \mathrm{~cm}$, mulher $\geq 88 \mathrm{~cm}$.
Tabela 5. Consumo alimentar antes e um ano após cirurgia bariátrica por derivação gástrica em Y de Roux, no período de 2010 a $2014(\mathrm{n}=50)$. Rio Grande do Sul, Brasil.

\begin{tabular}{|c|c|c|c|c|}
\hline Variáveis & Mínimo & Máximo & Média $\pm D P$ & p \\
\hline \multicolumn{5}{|l|}{ Kcal } \\
\hline Pré & 1860 & 3765 & $2761,98 \pm 480,63$ & \multirow{2}{*}{$0,002 *$} \\
\hline Pós & 1220 & 1990 & $1651,50 \pm 158,89$ & \\
\hline \multicolumn{5}{|l|}{ HC (\%) } \\
\hline Pré & 47 & 71 & $55,28 \pm 7,12$ & \multirow{2}{*}{$0,730^{+}$} \\
\hline Pós & 47 & 66 & $55,56 \pm 3,53$ & \\
\hline \multicolumn{5}{|l|}{ PTN (\%) } \\
\hline Pré & 10 & 32 & $19,88 \pm 7,58$ & \multirow{2}{*}{$0,312^{+}$} \\
\hline Pós & 14 & 22 & $18,62 \pm 1,76$ & \\
\hline \multicolumn{5}{|l|}{$\begin{array}{l}\text { PTN } \\
\text { (g/kg peso atual) }\end{array}$} \\
\hline Pré & 0,50 & 1,90 & $1,07 \pm 0,44$ & \multirow{2}{*}{$0,501^{\dagger}$} \\
\hline Pós & 0,70 & 1,50 & $1,02 \pm 2,88$ & \\
\hline \multicolumn{5}{|l|}{ LIP (\%) } \\
\hline Pré & 16 & 38 & $24,96 \pm 5,80$ & \multirow{2}{*}{$0,523^{+}$} \\
\hline Pós & 17 & 33 & $25,22 \pm 2,88$ & \\
\hline \multicolumn{5}{|l|}{ Cálcio (mg) } \\
\hline Pré & 04 & 786 & $428,64 \pm 228,37$ & \multirow{2}{*}{$0,761^{+}$} \\
\hline Pós & 04 & 780 & $402,09 \pm 171,40$ & \\
\hline \multicolumn{5}{|l|}{ Ferro (mg) } \\
\hline Pré & 0,08 & 15,64 & $10,70 \pm 4,01$ & \multirow{2}{*}{$0,138^{+}$} \\
\hline Pós & 0,09 & 19,40 & $10,86 \pm 5,17$ & \\
\hline \multicolumn{5}{|c|}{ Vitamina D (mcg) } \\
\hline Pré & 0,00 & 2,50 & $0,80 \pm 0,70$ & \multirow{2}{*}{$0,342^{+}$} \\
\hline Pós & 0,00 & 2,40 & $0,68 \pm 0,64$ & \\
\hline \multicolumn{5}{|c|}{ Vitamina B12 (mcg) } \\
\hline Pré & 0,02 & 5,74 & $2,38 \pm 1,60$ & \multirow{2}{*}{$<0,001^{\dagger}$} \\
\hline Pós & 0,00 & 4,70 & $1,72 \pm 1,30$ & \\
\hline
\end{tabular}

* Teste $t$ de Student.

† Teste Wilcoxon.

DP, desvio padrão; Kcal, quilocalorias; HC, carboidrato; PTN, proteína; LIP, lipídeo; mg, miligramas; mcg, microgramas; Pré, antes da cirurgia; Pós, um ano após a cirurgia. 
A Tabela 5 descreve o consumo alimentar dos pacientes, antes e após seguimento de um ano da realização da cirurgia bariátrica. Em média, a ingestão de calorias e de vitamina B12 diminuiu significativamente $(\mathrm{p}<0,05)$. As outras variáveis referentes ao consumo alimentar não apresentaram alteração significativa.

\section{DISCUSSÃO}

O aumento na prevalência de obesidade que vem ocorrendo nas últimas décadas envolve ambos os sexos e todas as classes sociais e níveis culturais [20]. No presente estudo, a maioria dos pacientes incluídos era do sexo feminino. Lehmann et al. [21] e Rangel et al. [20] que realizaram estudos com adultos que realizaram cirurgia bariátrica, também mostram dados com $76,6 \%$ e $75 \%$ do sexo feminino, respectivamente, em suas amostras. Essa maior proporção de mulheres entre as pessoas submetidas à cirurgia bariátrica pode ter várias explicações, desde a possibilidade de uma maior prevalência de obesidade no sexo feminino em determinadas populações, até a maior preocupação das mulheres com a saúde ou, mesmo, com a aparência física, tendo em vista os padrões estéticos vigentes na atualidade. Em relação à faixa etária, Quadros et al. [22] mostraram média de 40 anos, semelhante à deste estudo.

Em relação às comorbidades, $35(70 \%)$ dos pacientes avaliados apresentavam uma ou mais doenças antes da cirurgia bariátrica. Estudos mostraram a completa remissão de doenças prévias relacionadas à obesidade entre 82 a $98 \%$ dos operados, com normalização dos valores glicêmicos e pressão arterial sistêmica no período dos 12 meses após a cirurgia bariátrica [23-25].

Em relação à atividade física, na casuística deste estudo houve diminuição significativa do número de pacientes sedentários. O sedentarismo é um dos aspectos de estilo de vida que mais acometem a qualidade de vida dos obesos, sendo considerado fator de risco primário e independente para o desenvolvimento da obesidade, e cuja contribuição tem efeito cumulativo [26]. Comportamentos sedentários, como permanecer por várias horas na frente da televisão, computador, videogames, entre outros, indicam que nos dias atuais gastam-se menos calorias por dia do que há 100 anos atrás, o que explica o aparecimento de diversas doenças crônicas, além do stress que está relacionado à ausência do exercício físico regular [27]. Sendo assim, recomenda-se que após a cirurgia os pacientes sejam estimulados a aumentar as atividades físicas e consequentemente o gasto calórico. Por outro lado, a atividade física vai sendo facilitada à medida em que $\mathrm{o}$ peso corporal vai diminuindo, resultando em melhora da qualidade de vida e manutenção do peso.

Em relação ao consumo de álcool, este estudo não encontrou diferença significativa entre o momento prévio e o posterior à cirurgia. Normalmente os pacientes são orientados a evitar bebidas alcoólicas após a cirurgia bariátrica, uma vez que o álcool contém alta taxa calórica e pode causar danos às mucosas do aparelho digestivo, reduzindo a absorção de alguns nutrientes. Além disso, após esse tipo de procedimento cirúrgico a absorção do álcool torna-se muito rápida, mesmo quando consumido em pequenas quantidades, o que predispõe à embriaguez. As bebidas gaseificadas também são contraindicadas por causar dilatação gástrica [28].

Quanto aos exames bioquímicos no pós-operatório, este estudo observou que a maioria dos pacientes apresentou níveis séricos dentro da normalidade para vitamina $\mathrm{B} 12$, hematócrito, hemoglobina, albumina e ferritina, em exames que foram realizados entre 6 meses e um ano após a cirurgia. Apenas um paciente apresentou níveis séricos baixos de vitamina B12, o que pode estar relacionado à oferta insuficiente desse micronutriente na dieta, constatada não só nesse paciente como na média geral da amostra, já que o estudo identificou uma diminuição significativa de ingestão de vitamina B12 entre os períodos pré e pós-cirurgia.

A deficiência de vitamina B12, geralmente definida como níveis séricos inferiores a $200 \mathrm{pg} / \mathrm{mL}$, é referida na literatura como uma das mais comuns complicações metabólicas/nutricionais da cirurgia bariátrica, podendo surgir em um tempo variável após a cirurgia e relacionada ou não à oferta na alimentação. Vários fatores resultantes da cirurgia pela técnica de DGYR contribuem para o decréscimo da biodisponibilidade da vitamina B12, como alteração da anatomia do estômago, passagem dos alimentos diretamente ao duodeno e diminuição do tempo de contato dos alimentos com o íleo terminal. Com o uso dessa técnica, a deficiência de vitamina B12 é relatada em 12 a $75 \%$ dos pacientes, dependendo do tempo pós-operatório em que for avaliada. Pode ser vista a partir de seis meses de pós operatório, ou quando as reservas no fígado se esgotam. Estudos mostraram que 10 anos após o procedimento existia alta prevalência (mais de 70\%) de deficiência da vitamina B12 [29-32]. Na presente casuística, a deficiência não foi observada na grande maioria dos pacientes, possivelmente devido ao curto tempo (seis meses a um ano) de pós operatório 
em que os exames foram feitos, e em virtude de todos os pacientes terem recebido prescrição de suplemento polivitamínico após o procedimento.

A hipoalbuminemia após a DGYR pode variar de $13 \%$ em pacientes após dois anos de cirurgia [33] a 27,9\% após 10 anos [32]. Após oito meses de cirurgia, Farias et al. [34] encontraram concentrações de albumina normais. Já Skroubis et al. [35], após avaliar 243 pacientes, observaram baixa incidência de hipoalbuminemia e $37,7 \%$ dos pacientes apresentaram níveis baixos de ferritina após dois anos do procedimento cirúrgico. Neste estudo, no período analisado, não foi observada deficiência de albumina.

Três $(6 \%)$ dos pacientes deste estudo apresentaram deficiência de ferritina entre seis meses e um ano após a cirurgia. Anemia com baixos níveis de ferritina pode afetar dois terços dos pacientes submetidos à cirurgia bariátrica, pela deficiência de ferro na dieta. Em pacientes submetidos à DGYR, a ocorrência de anemia varia entre $20 \%$ a $49 \%$ [31,36,37]. Neste estudo, sete (14\%) pacientes apresentaram hemoglobina baixa e três $(6 \%)$ pacientes apresentaram hematócrito baixo. Santos [38] analisou 15 pacientes após seis meses da cirurgia por DGYR, e todos mantiveram seus valores de referência dentro do normal, sendo que a maioria recebeu suplementação de ferro.

A quantidade de ferro elementar presente nos polivitamínicos geralmente é pequena (10 a $20 \mathrm{mg}$ por comprimido), sendo considerada insuficiente para evitar a deficiência de ferro em pacientes submetidos à DGYR. As recomendações atuais para a prevenção da deficiência de ferro incluem a administração de 40 a $80 \mathrm{mg}$ de ferro elementar por dia (200 a $400 \mathrm{mg}$ de sulfato ferroso). Nas mulheres em idade reprodutiva, as recomendações aumentam para 80 a $160 \mathrm{mg}$ de ferro elementar por dia (400 a $800 \mathrm{mg}$ de sulfato ferroso) [39-42]. Dessa forma, história clínica de anemia, alterações nos valores laboratoriais, idade, sexo e aspectos reprodutivos devem ser analisados antes de prescrever o composto com ferro.

$\mathrm{Na}$ análise da perda de peso, identificou-se redução de $31,92 \%$ neste estudo. Outro estudo, com 250 pacientes avaliados após o primeiro ano de cirurgia, mostrou redução de $37,5 \%$ no peso [43]. Em contrapartida, Quadros et al. [22] encontraram resultados inferiores em um igual período de tempo $(30,69 \%)$. Outras pesquisas mostraram que a redução do peso é maior no primeiro semestre, tornando-se após lenta e contínua, atingindo perda de 35 a $40 \%$ do peso inicial entre o primeiro e segundo ano após a realização da cirurgia [44].
Com relação à redução no IMC, os dados do presente estudo são semelhantes aos encontrados por outros pesquisadores. Carvalho et al. [44] e Santos

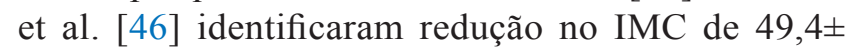
$7,4 \mathrm{~kg} / \mathrm{m}^{2}$ para $32,9 \pm 4,98 \mathrm{~kg} / \mathrm{m}^{2}$ e de $50,4 \pm 7,9 \mathrm{~kg} / \mathrm{m}^{2}$ para $31,9 \pm 5,85 \mathrm{~kg} / \mathrm{m}^{2} \mathrm{em}$ um ano após a cirurgia. Neste estudo também se observou uma redução do IMC, de $43,55 \pm 5,53 \mathrm{~kg} / \mathrm{m}^{2}$ antes para $29,55 \pm 4,22 \mathrm{~kg} / \mathrm{m}^{2}$ após a cirurgia.

A diminuição de 5 a $10 \%$ do peso corporal e a diminuição da CA produzem efeitos positivos na síndrome metabólica e melhora de algumas doenças relacionadas à obesidade. A cirurgia bariátrica é um dos métodos mais eficazes na perda e manutenção do peso, levando a uma perda de 20 a $70 \%$ do excesso de peso e consequentemente a uma melhora nítida dos componentes da síndrome metabólica [47]. Entretanto, vitaminas e minerais são fatores e cofatores essenciais em muitos processos biológicos que regulam o peso corporal direta ou indiretamente, e por isso não devem ser negligenciados. Os benefícios metabólicos desses micronutrientes no controle da perda de peso incluem a regulação do apetite e a absorção de nutrientes. Assim, o adequado consumo de micronutrientes é importante não só para a manutenção dos processos metabólicos do organismo, mas também para obter sucesso na manutenção e na perda de peso a longo prazo [11].

A ingestão suficiente de micronutrientes a partir da alimentação é a forma mais natural de manter as reservas em níveis desejáveis. Porém, pacientes que realizam a cirurgia bariátrica necessitam de uma dosagem diária de polivitamínico/mineral para garantir um aporte adequado. Portanto, tem sido recomendado iniciar a suplementação logo após a alta hospitalar [11]. Seria importante conhecer os resultados dos exames bioquímicos (vitamina B12, hemoglobina, hematócrito, albumina, ferritina, cálcio e vitamina $\mathrm{D}$ ) prévios à cirurgia bariátrica, pois as deficiências encontradas já podem começar a ser corrigidas antes da cirurgia. A suplementação nutricional é muito enfatizada como importante conduta terapêutica para o sucesso do tratamento cirúrgico da obesidade [48]. A utilização do suplemento nutricional só é considerada correta quando utilizada pelo menos cinco vezes por semana [15], mas infelizmente apenas $33 \%$ dos pacientes atendem a essa recomendação. Além disso, 7,7\% dos pacientes deixam de utilizar os polivitamínicos/minerais após dois anos de cirurgia [30].

Concluindo, os pacientes após cirurgia bariátrica por DGYR tiveram uma perda de peso efetiva, com redução do IMC e da CA. A taxa de sedentarismo diminuiu. A análise do consumo alimentar mostrou que 
a ingestão de calorias diminuiu entre a avaliação inicial e a realizada um ano após a cirurgia. A ingestão média de nutrientes não sofreu alteração, com exceção da ingestão de vitamina B12, cuja média diminuiu, sendo que um paciente apresentou deficiência sérica dessa vitamina e sete pacientes apresentaram anemia após a cirurgia. Pode-se concluir que a cirurgia por DGYR nos pacientes estudados foi efetiva para o tratamento da obesidade, e que há necessidade de acompanhamento do estado nutricional a longo prazo, para garantir o sucesso do tratamento e evitar deficiência de nutrientes.

\section{NOTA}

Declaração de conflitos de interesse

Os autores declaram não haver conflitos de interesse relevantes ao conteúdo deste estudo.

\section{REFERÊNCIAS}

1. World Health Organization. Global status report on noncommunicable diseases 2014. Geneva: WHO; 2014.

2. Ministério da Saúde (BR). VIGITEL, Brasil 2014: vigilância de fatores de risco e proteção para doenças crônicas por inquérito telefônico. Brasília: Ministério da Saúde; 2015.

3. Associação Brasileira para Estudos da Obesidade e da Síndrome Metabólica. Quase $60 \%$ dos brasileiros estão acima do peso, revela IBGE [Internet]. 2015 [cited 2016 July 14]. Available from: http://www.abeso.org.br/noticia/quase-60-dos-brasileiros-estao-acima-dopeso-revela-pesquisa-do-ibge

4. Instituto Brasileiro de Geografia e Estatística. Pesquisa de Orçamentos Familiares 2008-2009: análise do consumo alimentar pessoal no Brasil. Rio de Janeiro: IBGE; 2011.

5. Bressan J, Costa AGV. Tratamento nutricional da obesidade. In: Nunes MA, Appolinário JC, Galvão AL, Coutinho W, et al. Transtornos Alimentares e Obesidade. 2a ed. Porto Alegre: Artmed; 2006. p. 315-325.

6. Fontaine KR, Redden DT, Wang C, Westfall AO, Allison DB. Years of life lost due extremely obesity. JAMA. 2003 Jan 8;289(2):187-93. http://dx.doi.org/10.1001/jama.289.2.187

7. Sjöström L, Gummesson A, Sjöström CD, Narbro K, Peltonen M, Wedel H, Bengtsson C, Bouchard C, Carlsson B, Dahlgren S, Jacobson P, Karason K, Karlsson J, Larsson B, Lindroos AK, Lönroth H, Näslund I, Olbers T, Stenlöf K, Torgerson J, Carlsson LM; Swedish Obese Subjects Study. Effects of bariatric surgery on mortality in Swedish obese subjects. Lancet Oncol. 2009 July;10(7):653-62. http://dx.doi. org/10.1016/S1470-2045(09)70159-7

8. Sociedade Brasileira de Cirurgia Bariátrica e Metabólica. Número de Cirurgias Bariátricas realizadas no Brasil [Internet]. [cited 2016 July 14]. Available from: http://www.sbcb.org.br/imprensa.php?menu=3

9. Parkes E. Nutritional management of patients after bariatric surgery. Am J Med Sci. 2006; 331:207-13. http://dx.doi.org/10.1097/00000441200604000-00007

10. Gong K, Gagner M, Pomp A, Almahmeed T, Bardaro SJ. Micronutrient deficiencies after laparoscopic gastric bypass: recommendations. Obes Surg. 2008;18:1062-66. http://dx.doi.org/10.1007/s11695-008-9577-9

11. Allied Health Sciences Section Ad Hoc Nutrition Committee, Aills L, Blankenship J, Buffington C, Furtado M, Parrott J. ASMBS Allied Health Nutritional Guidelines for the Surgical Weight Loss Patient. Surg Obes Relat Dis. 2008 Sept-Oct;4(5 Suppl):S73-108. http://dx.doi. org/10.1016/j.soard.2008.03.002

12. Pedrosa IV, Burgos MGPA, Souza NC, Morais CN. Aspectos nutricionais em obesos antes e após a cirurgia bariátrica. Rev Col Bras Cir. 2009;36(4):316-22. http://dx.doi.org/10.1590/S0100-69912009000400008

13. Beleli CAV, Filho AC, Silva RM, Camargo MA, Scopin DR. Fatores preditivos na perda ponderal de pacientes submetidos ao Bypass Gástrico em Y-de-Roux. BMI. 2011;1:16-23.

14. Dalcanale L, Oliveira CP, Faintuch J, Nogueira MA, Rondó P, Lima VM, Mendonça S, Pajecki D, Mancini M, Carrilho FJ. Long-term nutritional outcome after gastric bypass. Obes Surg. 2010 Feb;20(2):181-7. http://dx.doi.org/10.1007/s11695-009-9916-5

15. World Health Organization. Global status report on noncommunicable diseases 2010 - Description of the global burden of NCDs, their risk factors and determinants. Geneva: WHO; 2011.

16. World Health Organization. Waist circumference and waist-hip ratio: report of WHO expert consultation. Geneva: WHO; 2011.

17. Institute of Medicine. Dietary Reference intake for thiamin, riboflavin, niacin, vitamin B6, folate, vitamin B12, pantothenic acid, biotin and choline. Washington: National Academy Press; 1998.

18. Institute of Medicine. Dietary Reference Intakes for Water, Potassium, Sodium, Chloride, and Sulfate. Washington D.C.: National Academy Press; 2004.

19. Resende LMH, Viana LG, Vidigal PG. Protocolos clínicos dos exames laboratoriais: versão preliminar. Belo Horizonte: UFMG; 2009.

20. Rangel LOB, Faria VSP, Magalhães EA, Araújo ACT, Bastos EMRD. Perfil de saúde e nutricional de pacientes portadores de obesidade mórbida candidatos à cirurgia bariátrica. Rev Bra Nutr Clin. 2007;22:214-9.

21. Lehmann ALF, Valezi AC, Brito EM, Marson AC, Souza JCL. Correlação entre hipomotilidade da vesícula biliar e desenvolvimento de colecistolitíase após operação bariátrica. Rev Col Bras Cir. 2006;33:285-8. http://dx.doi.org/10.1590/S0100-69912006000500005 
22. Quadros MRR, Savaris AL, Ferreira MV, Branco Filho AJ. Intolerância alimentar no pós-operatório de pacientes submetidos à cirurgia bariátrica. Rev Bra Nutr Clin. 2007;22:15-9.

23. Martins, MVDC, Souza AAPS. Mecanismos cirúrgicos de controle do diabetes mellitus tipo 2 após cirurgia bariátrica. Rev Col Bras Cir. 2007;34:343-6. http://dx.doi.org/10.1590/S0100-69912007000500013

24. Diniz MFHS, Passos VMA, Barreto SM, Diniz MTC, Linares DB, Mendes LN. Perfil de pacientes obesos classe III do Sistema Público de Saúde submetidos à gastroplastia em "Y de ROUX", no Hospital das Clínicas da UFMG: altas prevalências de superobesidade, co-morbidades e mortalidade hospitalar. Rev Med Minas Gerais. 2008;18:183-90.

25. Lima PAL, Barreto Filho JAS, Oliveira MHA, Almeida F, Moura JS, Alves Junior A. Avaliação dos níveis plasmáticos de IGF-1, glicose e insulina no pré e pós-operatório de pacientes submetidos à cirurgia de fobi-capella. J Port Gastrenterol. 2008;15:196-203.

26. De Zwaan M, Mitchell JE, Howell LM, Monson N, Swan-Kremeier L, Roerig JL, Kolotkin RL, Crosby RD. Two measures of health-related quality of life in morbid obesity. Obes Res. 2002 Nov;10(11):1143-51. http://dx.doi.org/10.1038/oby.2002.155

27. Ishitani LH; Franco GC; Perpétuo IHO; França E. Desigualdade social e mortalidade precoce por doenças cardiovasculares no Brasil Rev Saúde Pública. 2006;4(4): 684-91. http://dx.doi.org/10.1590/S0034-89102006000500019

28. Galvão TD. Consumo de bebida alcoólica após a cirurgia bariátrica [Internet]. 2014 [cited 2015 Sept 20]. Available from: http:/www. drthalesdelmondes.com.br/consumo-de-bebida-alcoolica-apos-a-cirurgia-bariatrica

29. Malinowski SS. Nutritional and metabolic complications of bariatric surgery. Am J Med Sci. 2006;331:219-25. http://dx.doi. org/10.1097/00000441-200604000-00009

30. Slater GH, Ren CJ, Siegel N, Williams T, Barr D, Wolfe B, Dolan K, Fielding GA. Serum fat-soluble vitamin deficiency and abnorma calcium metabolism after malabsorptive bariatric surgery. J Gastrointest Surg. 2004 Jan;8(1):48-55; discussion 54-5. http://dx.doi. org/10.1016/j.gassur.2003.09.020

31. Brolin RE, Gorman JH, Gorman RC, Petschenik AJ, Bradley LJ, Kenler HA, Cody RP. Are vitamin B12 and folate deficiency clinically important after roux-en-Y gastric bypass? J Gastrointest Surg. 1998 Sept-Oct;2(5):436-42. http://dx.doi.org/10.1016/S1091255X(98)80034-6

32. Higa K, Ho T, Tercero F, Yunus T, Boone KB. Long-term results after laparoscopic roux-en-Y gastric bypass: 10-year follow-up. Surg Obes Relat Dis. 2011 July-Aug;7(4):516-25. http://dx.doi.org/10.1016/j.soard.2010.10.019

33. Mango VL, Frishman WH. Physiologic, psychologic, and metabolic consequences of bariatric surgery. Cardiol Rev. 2006 Sept-Oct; 14(5):232-7. http://dx.doi.org/10.1097/01.crd.0000223656.06812.ae

34. Farias LM, Coêlho MPSS, Barbosa RF, Santos GS, Marreiro DN. Aspectos nutricionais em mulheres obesas submetidas à gastroplastia vertical com derivação gastro-jejunal em Y-de-Roux. Rev Bra Nutr Clin. 2006;21:98-103.

35. Skroubis G, Sakellaropoulos G, Pouggouras K, Mead N, Nikiforidis G, Kalfarentzos F. Comparison of nutritional deficiencies after Rouxen- Y gastric bypass and after biliopancreatic diversion with Rouxen-Y gastric bypass. Obes Surg. 2002;12:551-8. http://x.doi. org/10.1381/096089202762252334

36. Amaral JF, Thompson WR, Caldwell MD, Martin HF, Randall HT. Prospective hematologic evaluation of gastric exclusion surgery for morbid obesity. Ann Surg. 1985;201(2):186-93. http://dx.doi.org/10.1097/00000658-198502000-00009

37. Brolin RE, Gorman RC, Milgrim LM, Kenler HA. Multivitamin prophylaxis in prevention of post-gastric bypass vitamin and mineral deficiencies. Int J Obes. 1991;15(10):661-7.

38. Santos LA. Avaliação nutricional de pacientes obesos antes e seis meses após a cirurgia bariátrica [Dissertação]. Belo Horizonte: Universidade Federal de Minas Gerais; 2007

39. Parkes E. Nutritional management of patients after bariatric surgery. Am J Med Sci. 2006;331(4):207-13. http://dx.doi.org/10.1097/00000441200604000-00007

40. Malinowski SS. Nutritional and metabolic complications of bariatric surgery. Am J Med Sci. 2006;331(4):219-25. http://dx.doi. org/10.1097/00000441-200604000-00009

41. Fujioka K. Follow-up of nutritional and metabolic problems after bariatric surgery. Diabetes Care. 2005;28(2):481-4. http://dx.doi. org/10.2337/diacare.28.2.481

42. Trost LB, Bergfeld WF, Calogeras E. The diagnosis and treatment of iron deficiency and its potential relationship to hair loss. J Am Acad Dermatol. 2006;54:824-44. http://dx.doi.org/10.1016/j.jaad.2005.11.1104

43. Valezi AC, Júnior, JM, Brito EM, Marson AC. Gastroplastia vertical com bandagem em Y-de-roux: análise de resultados. Rev Col Bras Cir. 2004;31(1):49-56. http://dx.doi.org/10.1590/S0100-69912004000100010

44. Garrido Júnior AB, Ferraz EM, Barroso FL, Marchesini JB, Szegö T. Cirurgia da obesidade. São Paulo: Atheneu Editora; 2013.

45. Carvalho PS, Moreira CLCB, Barelli MC, Oliveira FH, Guzzo MF, Miguel GP, Zandonade E. Cirurgia bariátrica cura síndrome metabólica? Arq Bras Endocrinol Metab. 2007;51:79-85. http://dx.doi.org/10.1590/S0004-27302007000100013

46. Santos EMC, Burgos MGPA, Silva SA. Perda ponderal após cirurgia bariátrica de Fobi-Capella: realidade de um hospital universitário do nordeste brasileiro. Rev Bra Nutr Clin. 2006;21:188-92.

47. Sociedade Brasileira de Cardiologia. I diretriz brasileira de diagnóstico e tratamento da síndrome metabólica. Rio de Janeiro: Sociedade Brasileira de Cardiologia; 2005.

48. Mechanick JI, Kushner RF, Sugerman HJ, Gonzalez-Campoy JM, Collazo-Clavell ML, Guven S, Spitz AF, Apovian CM, Livingston EH, Brolin R, Sarwer DB, Anderson WA, Dixon J. American Association of Clinical Endocrinologists, The Obesity Society, and American Society for Metabolic \& Bariatric Surgery Medical Guidelines for Clinical Practice for the perioperative nutritional, metabolic, and nonsurgical support of the bariatric surgery patient. Surg Obes Relat Dis. 2008 Sept-Oct;4(5 Suppl):S109-84. http://dx.doi.org/10.1016/j. soard.2008.08.009 